Artículo

\title{
Caracterización molecular de caimito en el estado de Morelos
}

\author{
María Andrade-Rodríguez ${ }^{1}$ \\ Teresa de J. Rodríguez-Rojas ${ }^{2 \S}$ \\ Oscar G. Villegas-Torres ${ }^{1}$ \\ Antonio Castillo Gutiérrez ${ }^{2}$ \\ ${ }^{1}$ Posgrado en Ciencia Agropecuarias y Desarrollo Rural-Facultad de Ciencias Agropecuaria-Universidad \\ Autónoma del Estado de Morelos. Avenida Universidad 1001, Colonia Chamilpa, Cuernavaca, Morelos, \\ México. CP. 62209. Tel. 777 3702946. ${ }^{2}$ Escuela de Estudios Superiores de Xalostoc-Universidad Autónoma \\ del Estado de Morelos. Av. Nicolás Bravo s/n, Parque Industrial Cuautla, Xalostoc, Ayala, Morelos, \\ México. CP. 62717. Tel. 777 3297981, 777 329-7988. \\ §Autor para correspondencia: teresa.rodriguez@uaem.mx.
}

\section{Resumen}

La fruticultura de México cuenta con 63 especies comerciales y 220 especies con potencial alimenticio. En el estado de Morelos se han reportado 1.7 ha de caimito en los municipios de Coatlán del Río y Tetecala de la Reforma. El caimito es una fuente alternativa para la diversificación de la agricultura, así como para la exportación frutícola. El conocimiento de la variabilidad genética de las especies permitirá el desarrollo de programas orientados a la conservación de germoplasma de forma in situ o ex situ, programas de certificación, así como proporcionar a los productores una amplia selección de plantas. El objetivo de la investigación fue caracterizar molecularmente árboles de caimito seleccionados previamente en Coatlán del Río y Tetecala de la Reforma, Morelos, México, utilizando la técnica de RAPDs. Se seleccionaron 13 árboles de caimito tomando en cuenta para la selección con base en las características morfológicas y organolépticas. Se realizó el aislamiento de ADN genómico y se utilizaron 30 iniciadores RAPD. Los datos moleculares se procesaron utilizando el sistema de análisis multivariado y taxonómico numérico (NTSYSpc 2.1). La metodología utilizada permitió caracterizar nueve árboles. El análisis del conglomerado obtenido muestra que a un nivel de 0.71 de distancia genética se forman cuatro agrupaciones. Los árboles 14 y 15 no comparten similitud genética con los otros árboles y estos fueron a su vez los que mostraron mayor número de bandas marcadoras y así como también fueron unos de los que destacaron por sabor.

Palabras clave: caracterización de árboles frutales, extracción de ADN, marcadores moleculares, RAPDs.

Recibido: abril de 2021

Aceptado: agosto de 2021 


\section{Introducción}

La fruticultura en México cuenta con 63 especies comerciales y 220 especies con potencial alimenticio, algunas especies aún no se reflejan en las estadísticas ya que se cultivan localmente en huertos familiares o son de recolección (Borys y Borys, 2001), entre las cuales están las de la familia Sapotaceae; de esta, el caimito (C. cainito L.) es originario de Centroamérica e islas del Caribe y las Antillas. En el estado de Morelos se han reportado 1.7 ha de caimito en los municipios de Coatlán del Río y Tetecala de la Reforma (Álvarez-Vargas et al., 2006; SIACON, 2018).

Las especies de Chrysophyllum L. (Sapotaceae) se propagan ampliamente como plantas ornamentales debido a su densidad follaje colorido y por sus frutos comestibles (El-Harwary et al., 2019). Son frutos carnosos, tipo baya globosa o redondeada, la pulpa es carnosa, destaca por su exquisito sabor y valor nutritivo, ya que los frutos son ricos en carbohidratos, calcio y fósforo, se pueden consumir como fruto fresco, en jugos o ensaladas, así como también se utilizan con fin ornamental por su porte y color dorado del envés de las hojas (Morton, 1987; Pennington, 1990; Rojas-Rodríguez y Torres-Córdoba, 2012).

Es por lo antes mencionado que Azurdia et al. (1995) sugiere la importancia del caimito como fuente alternativa para la diversificación de la agricultura, así como para la exportación frutícola. Es poco el conocimiento que se tiene sobre las técnicas de propagación sexual y asexual de este frutal, así como sobre su cultivo en general, con condiciones y prácticas que aseguren una buena producción de frutos de alta calidad. Para lograr esto se necesita producir portainjertos adecuados con el fin de perpetuar las variedades o tipos seleccionados de esta planta (Álvarez et al., 2004).

Al respecto Crane y Balerdi (2019) mencionan que el caimito puede propagarse por semilla, estaca e injerto, los árboles que provienen de semilla dan fruto en 5 a 10 años, mientras que árboles de propagación vegetativa pueden comenzar la producción de frutos en 1 a 2 años y en el injerto se ha reportado que caimito produce árboles enanos de crecimiento lento. La propagación asexual limita el número de generaciones que han pasado desde su primer cultivo por parte de los humanos y el grado de evolución que ha ocurrido bajo la selección humana en estos taxones (Zohary y SpiegelRoy, 1975; Clement, 1989). Al propagarse el caimito por semilla genera una amplia diversidad genética, por ello es recomendable multiplicar los árboles seleccionados por su capacidad productiva y calidad de sus frutos, mediante la injertación (Avilan et al., 1992).

Si distintas variedades cultivadas se seleccionan por fenotipos contrastantes, la varianza fenotípica será mayor en cultivos plantas (Parker et al., 2010). El conocimiento de la variabilidad genética de las especies permitirá el desarrollo de programas orientados a la conservación de germoplasma de forma in situ o ex situ, programas de certificación, así como proporcionar a los productores una amplia selección de plantas (Azurdia et al., 1997; Carrara, 2004; Nascimento et al., 2008). Existen diferentes métodos para caracterizar, identificar plantas especies, evaluar su diversidad y filogenia, aquellos basados en la morfología son los más utilizados (Bayuelo-Jiménez et al., 2006).

Los primeros estudios de caracterización de sapotáceas utilizaron caracteres morfológicos, fenológicos, y agronómicos; sin embargo, la clasificación con base en estos caracteres puede ser confusa porque los mismos son afectados por factores ambientales. El uso de isoenzimas puede ser de mayor utilidad; al respecto, fueron aplicadas para estudiar in situ 246 tipos de M. sapota y 287 
de C. cainito en Nicaragua (Benavides, 1998; Weaver, 1993) mediante caracterización morfológica registró una nueva especie de caimito llamada Micropholis chrysophylloides en Puerto Rico; sin embargo, éstas pueden ser afectadas por el ambiente y la etapa fenológica de la planta.

También se ha generado un gran número de métodos para identificar y analizar marcadores moleculares entre los que destacan los basados en la reacción en cadena de la polimerasa (PCR), que permiten amplificar secuencias específicas o al azar del ADN y requieren de pequeñas cantidades iniciales del mismo (Orona et al., 2006) no son afectados por el medio ambiente y pueden analizarse en cualquier etapa de desarrollo del cultivo, los marcadores RAPD son simples, eficientes, económicos, rápidos y discriminan eficientemente entre genotipos (Yasmin et al., 2006).

Marcadores como el citogenético y molecular permiten analizar las diferencias entre cromosomas, proteínas o ADN (Avise, 2004). Otra técnica empleada es la secuenciación del gen del cloroplasto $n d h \mathrm{~F}$ para estudiar la filogenia en la familia (Anderberg y Swensony, 2003). Las secuencias simples repetidas (SSR) fueron utilizadas para el análisis de la variabilidad del DNA del cloroplasto en Manilkara hubery (Rennó et al., 2008). Los RAPDs fueron utilizados para determinar el efecto de las condiciones ambientales en el genotipo de Manilkara sapota (Heaton et al., 1999) y determinar la estructura genética espacial de Chrysophyllum sanguinolentum (Degen et al., 2001), así como estudiar la diversidad genética de Vitellaria paradoxa (Fontaine et al., 2004).

En Pouteria sapota también se han usado los AFLP para estudiar la variación genética de selecciones cultivada (Carrara, 2004). Majourhat et al. (2008), utilizaron los RAPDs y las SSR para caracterizar morfotipos de Argania spinosa y observaron que los RAPDs presentaron alto polimorfismo y mayor cantidad de información que las SSR. Actualmente existe un gran número de colecciones de germoplasma con genotipos de un alto valor agronómico que podrían ser usados como progenitores en los programas de mejoramiento genético; sin embargo, en muchas ocasiones se desconoce su grado de diversidad y la relación existente entre materiales, lo que dificulta su utilización (Becerra y Paredes, 2000).

Los marcadores moleculares se utilizan ampliamente para implementar programas de mejoramiento y manejo de cultivos (Xin-hua et al., 2007). Este método es beneficioso para los ecologistas y conservacionistas al permitir la identificación de muestras (Hebert y Gregory, 2005). González-Hernández et al. (2012) hacen referencia que, aunque el chicozapote (Sapotaceae) es una especie económicamente importante en México, no hay información que permita generar programas apropiados para su mejoramiento y manejo de cultivo.

Debido a la escasa información acerca de caimito en cuanto a caracterización molecular que permita identificar a los árboles con mejores características que pudieran usarse para conservarlos y eventualmente integrarlos a un programa de mejoramiento genético a largo plazo o propagación vegetativa que permita tener huertas de caimito establecidas de manera homogénea y con frutos de calidad, el objetivo de la presente investigación fue caracterizar molecularmente árboles de caimito seleccionados previamente en Coatlán del Río y Tetecala de la Reforma, Morelos, México, utilizando la técnica de RAPDs, para poder determinar si existe una diferenciación genética de árboles que permita la identificación y selección de germoplasma sobresaliente; a través, de marcadores moleculares. 


\section{Materiales y métodos}

Se seleccionaron 13 árboles de caimito tomando en cuenta para la selección con base en las características morfológicas y organolépticas como el sabor del fruto, mayor rendimiento, tamaño y calidad, mediante entrevistas con los productores realizadas por Álvarez-Vargas et al. (2006) en Coatlán del Río y Tetecala de la Reforma, Morelos, México. Las características por las que se seleccionaron son porque los frutos de caimito son de color rojo, opaco y oscuro, existe gran variación en el matiz y cromaticidad de los frutos, probablemente por la amplia variedad en los colores y su intensidad. Las dimensiones del fruto, como largo y diámetro, muestran una variación alta con rangos de 60.3 y $54.1 \mathrm{~cm}$, respectivamente.

El 60\% del peso total del fruto corresponde a la cáscara, 3\% a la semilla y $37 \%$ a la pulpa, la cáscara del fruto está compuesta de un epicarpio delgado y duro, así como parte del mesocarpio que en la madurez comercial forma una cáscara resistente, la otra parte del mesocarpio es morada o blanca, acuosa y dulce y representa la pulpa del fruto. La gran variación en estos caracteres hace posible la búsqueda de materiales con mayor proporción de pulpa, para incrementar su uso potencial como fruta fresca o procesada. El caimito puede tener hasta ocho semillas, así como frutos sin semilla.

Los frutos de caimito presentan gran variación en características químicas como los sólidos solubles, azúcares totales. Los valores promedio de sólidos solubles son de 10.1 Brix e indican que los frutos proporcionan buena cantidad de energía a quien los consuma, algunos frutos alcanzan valores de $185.9 \mathrm{mg} \mathrm{g}^{-1}$ de azúcares totales. Actualmente, se han realizado pocos estudios de calidad para determinar las características del fruto que sean atractivas para el consumidor; sin embargo, se prefieren frutos grandes, con pocas semillas y gran concentración de azúcares totales (Álvarez-Vargas et al., 2006). Los árboles fueron 1, 2, 3, 4, 5, 9, 10, 13, 14, 15, 17, 18 y 19.

\section{Colecta de hojas}

Se colectaron muestras de hojas jóvenes y sanas de 13 árboles de caimito seleccionados previamente. Las hojas fueron colocadas en bolsas de plástico, marcadas con la clave de cada árbol y transportadas, a temperatura ambiente al laboratorio. Las hojas fueron lavadas y se eliminó el agua de la superficie, posteriormente se pesaron $150 \mathrm{mg}$ de hoja de cada árbol y se congelaron a $-20^{\circ} \mathrm{C}$ hasta la extracción del ADN.

\section{Aislamiento del DNA genómico}

Se usaron muestras de $150 \mathrm{mg}$ de hojas de cada uno de los 13 árboles de caimito para extraer el ADN de acuerdo con la metodología reportada por Andrade-Rodríguez et al. (2005) sustituyendo el alcohol isoamilico por octanol. En el último paso de la metodología de extracción, el ADN obtenido se disolvió en $25 \mu \mathrm{l}$ de $0.1 \mathrm{TE}$ ( $1 \mathrm{mM}$ Tris- $\mathrm{HCl} \mathrm{pH} 8,0.1 \mathrm{mM}$ EDTA) con $20 \mathrm{ng}^{-1} \mathrm{l}^{-1} \mathrm{de}$ ARNasa, calentado a $37^{\circ} \mathrm{C}$ por $40 \mathrm{~min}$. Y almacenado a $-20{ }^{\circ} \mathrm{C}$ hasta su utilización.

La integridad del ADN de todos los árboles se estimó mediante electroforésis en gel de agarosa (ultrapura GIBCO) al 1\%, para lo cual se usó $1 \mu \mathrm{l}$ de muestra de ADN. La electroforesis se realizó a temperatura ambiente en buffer TAE (0.04 M tris-base, ácido acético glacial, 1 mM EDTA) a 75 voltios durante dos horas. El gel se tiñó con bromuro de etidio $\left(1 \mu \mathrm{g} \mathrm{ml}^{-1}\right)$. Las muestras de ADN se visualizaron y se documentaron con un analizador de geles (Syngene GVM20). 
La concentración del ADN y su pureza fueron cuantificadas en un espectrofotómetro Genesys $6^{\circledR}$ de luz ultravioleta, para lo cual se preparó una dilución de 1:150 [5 $\mu 1$ de ADN de la muestra más $745 \mu \mathrm{l}$ de TE 10X (10 mM Tris $\mathrm{HCl} \mathrm{pH} 8,1 \mathrm{mM}$ EDTA pH 8)]. La concentración se determinó mediante la fórmula: $\left[\mathrm{ADN}\left(\mathrm{ng} \mu \mathrm{l}^{-1}\right)\right]=\left(\mathrm{DO}_{260}\right)(\mathrm{FD})\left(50 \mu \mathrm{g} \cdot \mu \mathrm{l}^{-1}\right)$. Donde: $\mathrm{DO}_{260}=$ densidad óptica de la solución de ADN leída a la longitud de onda de $260 \mathrm{~nm}$. FD= factor de dilución. $50 \mu \mathrm{g} \mu \mathrm{l}^{-1}=$ concentración de ADN determinada para un valor de 1 a 260 nm.

La pureza se evaluó como la proporción de las lecturas a longitudes de onda de 260 y $280 \mathrm{~nm}$ (260/280). Los valores entre 1.8 y 2 de densidad óptica (DO) indican alto grado de pureza. Valores menores a 1.8 indican contaminación de la muestra de ADN con proteínas y otros elementos absorbentes de luz UV. Mientras que valores mayores a 2 indican contaminación por cloroformo, fenol u otra sustancia orgánica. Una vez obtenida la lectura para cada muestra de ADN, se prepararon las soluciones de trabajo a $20 \mathrm{ng}^{-1} \mathrm{l}^{-1}$.

\section{Análisis RAPD}

Se utilizaron 30 iniciadores, 20 con las secuencias del kit B, el A-07, A-08 (Operon Technologies Inc.) y ocho con las secuencias diseñadas por Rodríguez-Rojas et al. (2012) para estudios con sapotaceas, todos con secuencia arbitraria de 10 nucleótidos, de estos iniciadores se seleccionaron 12 que presentaron el mayor número de bandas más brillantes (Cuadro 1).

Cuadro 1. Secuencia de ocho de los iniciadores utilizados en la caracterización molecular de árboles de caimito, diseñadas por Rodríguez-Rojas et al. (2012).

\begin{tabular}{cccccc}
\hline Iniciador & Clave & Secuencia de bases & Iniciador & Clave & Secuencia de bases \\
\hline 23 & SAP-01 & 5' ATG CGA ACC G 3' & 27 & SAP-05 & 5' TAT AGG CCC T 3' \\
24 & SAP-02 & 5' GAC ACA TCG G 3' & 28 & SAP-06 & 5' CCT ACT CCA G 3' \\
25 & SAP-03 & 5' TGG GAC CTC C 3' & 29 & SAP-07 & 5' TGG GAA TCC C 3' \\
26 & SAP-04 & 5' GGA GCT ACC T 3' & 30 & SAP-08 & 5' GCC CCT ACT A 3' \\
\hline
\end{tabular}

La mezcla de reacción consistió en $10 \mu \mathrm{l}$ de dNTPs (5 $\mu \mathrm{M}$ de cada dNTP), $2.5 \mu \mathrm{l}$ amortiguador PCR (10X), $1.5 \mu \mathrm{l} \mathrm{de} \mathrm{MgCl}_{2}$ (75 mM), $2 \mu \mathrm{l}$ de iniciador (20 pmol), $0.3 \mu \mathrm{l}$ DNA polimerasa nativa INVITROGEN (1.5 U), $4 \mu \mathrm{l}$ de ADN (80 ng) ajustando a un volumen de $25 \mu \mathrm{l}$ con $4.7 \mu \mathrm{l}$ de agua destilada deionizada estéril. La amplificación del ADN se hizo mediante la reacción en cadena de la polimerasa (PCR) en un termociclador Techne TC-412. El programa del termociclador consistió en un ciclo de predesnaturalización de $\mathrm{ADN}$ a $94{ }^{\circ} \mathrm{C}$ por 4 min, más 35 ciclos integrados por las etapas siguientes: 1 min a $94^{\circ} \mathrm{C}$ para separar las hélices de $\mathrm{ADN}, 1 \mathrm{~min}$ a $36^{\circ} \mathrm{C}$ para la alineación de iniciador, 2 min a $72{ }^{\circ} \mathrm{C}$ para la polimerización de ADN y un ciclo de extensión final de 10 min a $72{ }^{\circ} \mathrm{C}$.

La separación de productos amplificados se realizó por electroforesis en gel de agarosa ultrapura (INVITROGEN) al 1.5\% (p/v). La electroforesis se efectuó con amortiguador TAE 1X aplicando 75 voltios durante $4.5 \mathrm{~h}$. Los geles fueron teñidos con bromuro de etidio $\left(1 \mu \mathrm{g} \mathrm{L}^{-1}\right)$ para evidenciar las bandas de ADN, y posteriormente se fotografiaron con el fotodocumentador Syngene GVM20. El tamaño de los fragmentos de ADN, producidos por los RAPDs, se obtuvo utilizando el programa Gen tools versión 3.06 de Syngene. 
El análisis de los datos moleculares para la caracterización de las 13 selecciones de caimito se realizó mediante la comparación de patrones de bandas de ADN producidos por los 12 iniciadores RAPD utilizando el programa Gene tools Labworks $4^{\circledR}$. La presencia de marcadores RAPD se determinó mediante el análisis de los perfiles electroforéticos generados por cada uno de los genotipos de caimito. Para la construcción del dendrograma, los datos se analizaron con base en la presencia (indicada por 1) o la ausencia (indicada por 0) de fragmentos amplificados con los iniciadores decámeros usados, los datos se procesaron utilizando el sistema de análisis multivariado y taxonómico numérico (NTSYSpc 2.1). Los análisis de agrupamiento se realizaron sobre la relación de matrices con el método de ligamiento promedio aritmético de grupos de pares no ponderados, UPGMA, por sus siglas en inglés (Avise, 1994).

\section{Resultados y discusión}

Los iniciadores seleccionados fueron OPB $(01,04,05,06,07,08,09,10,11$ y 12), SAP-01 y SAP04, por presentar el mayor número de productos amplificados, con bandas más brillantes y mejor definidas. Estos iniciadores generaron 190 fragmentos RAPD que variaron de 254 a 3.407 pb. El $97 \%$ de los fragmentos fueron polimórficos y permitieron caracterizar a 9 de los 13 árboles estudiados $(1,3,5,10,13,14,15,17$ y 19) con 71 fragmentos marcadores. En los árboles 2, 4, 9 y 18 no se obtuvieron fragmentos marcadores que permitieran su caracterización con los iniciadores usados (Cuadro 2). Los iniciadores que generaron mayor número de marcadores fueron el OPB-06 y el OPB-10, este último permitió identificar mayor número de árboles (6) junto con el SAP-01 y SAP-04 (Figura 1). Por el contrario, el iniciador OPB-11 produjo sólo tres fragmentos los cuales caracterizaron a dos árboles. Ningún iniciador por sí sólo permitió caracterizar a todas las plantas.

Cuadro 2. Fragmentos amplificados y caracterización molecular mediante RAPDs de árboles de caimito en Morelos, México.

\begin{tabular}{|c|c|c|c|c|c|}
\hline Iniciador & $\begin{array}{l}\text { Fragmentos } \\
\text { amplificados }\end{array}$ & $\begin{array}{l}\text { Fragmentos } \\
\text { polimórficos }\end{array}$ & $\begin{array}{c}\text { Polimorfismo } \\
(\%)\end{array}$ & Marcadores (pb) & $\begin{array}{c}\text { Planta } \\
\text { caracterizada }\end{array}$ \\
\hline OPB 01 & 17 & 17 & 100 & p:1906 & 13 \\
\hline \multirow[t]{4}{*}{ GTTTCGCTCC } & & & & $\mathrm{p}: 1813$ & 1 \\
\hline & & & & $\mathrm{p}: 877$ & 13 \\
\hline & & & & a:1615 & 13 \\
\hline & & & & a:1044 & 13 \\
\hline OPB 04 GGA & 15 & 14 & 93 & p:1319 & 5 \\
\hline CTG GAGT & & & & p: 490 & 19 \\
\hline OPB 05 & 13 & 13 & 100 & $\mathrm{p}: 1339,1081,866$ & 14 \\
\hline \multirow[t]{2}{*}{ TGCGCCCTTC } & & & & $\mathrm{p}: 492$ & 10 \\
\hline & & & & a: 1265 & 14 \\
\hline OPB 06 & 17 & 16 & 94 & $\mathrm{p}: 1877,1628,1324$ & 15 \\
\hline \multirow{3}{*}{ TGCTCTGCCC } & & & & $1063,909,519$ & \\
\hline & & & & p: $622,548,495$ & 10 \\
\hline & & & & $\mathrm{a}: 1131,995$ & 15 \\
\hline OPB O7 & 16 & 16 & 100 & p:2140, 1396, 918, 496 & 14 \\
\hline GGTGACGCAG & & & & a: 1727 & 13 \\
\hline OPB 08 & 16 & 16 & 100 & p:1493, 1100, 969, 254 & 15 \\
\hline GTCCACACGG & & & & a:732 & 15 \\
\hline
\end{tabular}




\begin{tabular}{|c|c|c|c|c|c|}
\hline Iniciador & $\begin{array}{l}\text { Fragmentos } \\
\text { amplificados }\end{array}$ & $\begin{array}{l}\text { Fragmentos } \\
\text { polimórficos }\end{array}$ & $\begin{array}{c}\text { Polimorfismo } \\
(\%)\end{array}$ & Marcadores (pb) & $\begin{array}{c}\text { Planta } \\
\text { caracterizada }\end{array}$ \\
\hline OPB 09 & 13 & 13 & 100 & $\mathrm{p}: 1561,1403$ & 15 \\
\hline TGGGGGACTC & & & & $\begin{array}{c}\mathrm{p}: 1357,1026,626 \\
\mathrm{a} \cdot 1940\end{array}$ & $\begin{array}{l}17 \\
15\end{array}$ \\
\hline $\begin{array}{c}\text { OPB } 10 \\
\text { CTGCTGGGAC }\end{array}$ & 21 & 21 & 100 & $\begin{array}{c}\text { p:2726, 2446, } 2280 \\
1934,1762,1628, \\
1258,899,716,654\end{array}$ & 14 \\
\hline & & & & $\begin{array}{c}\mathrm{p}: 1208 \\
\mathrm{a}: 1114 \\
\mathrm{a}: 467\end{array}$ & $\begin{array}{l}17 \\
14 \\
13\end{array}$ \\
\hline OPB 11 & 12 & 12 & 100 & p: 1187 & 10 \\
\hline GTAGACCCGT & & & & p:987, 831 & 15 \\
\hline OPB 12 & 12 & 11 & 91 & p:1192, 693, 596 & 14 \\
\hline CCTTGACGCA & & & & a:1028, 665 & 14 \\
\hline $\begin{array}{c}\text { SAP 01 } \\
\text { ATGCGAACCG }\end{array}$ & 21 & 20 & 95 & $\begin{array}{l}\text { p:669 } \\
\text { p:588 }\end{array}$ & $\begin{array}{c}3 \\
10\end{array}$ \\
\hline & & & & $\mathrm{p}: 1884, \frac{1689,1406}{960}$ & 14 \\
\hline SAP 04 & 17 & 16 & 94 & $\mathrm{p}: 310$ & 13 \\
\hline GGAGCTACCT & & & & p:687, 306 & 14 \\
\hline & & & & $\mathrm{p}: 1147$ & 15 \\
\hline & & & & a:599 & 13 \\
\hline
\end{tabular}

$\mathrm{Pb}=$ pares de bases; $\mathrm{a}=$ ausencia; $\mathrm{p}=$ presencia.

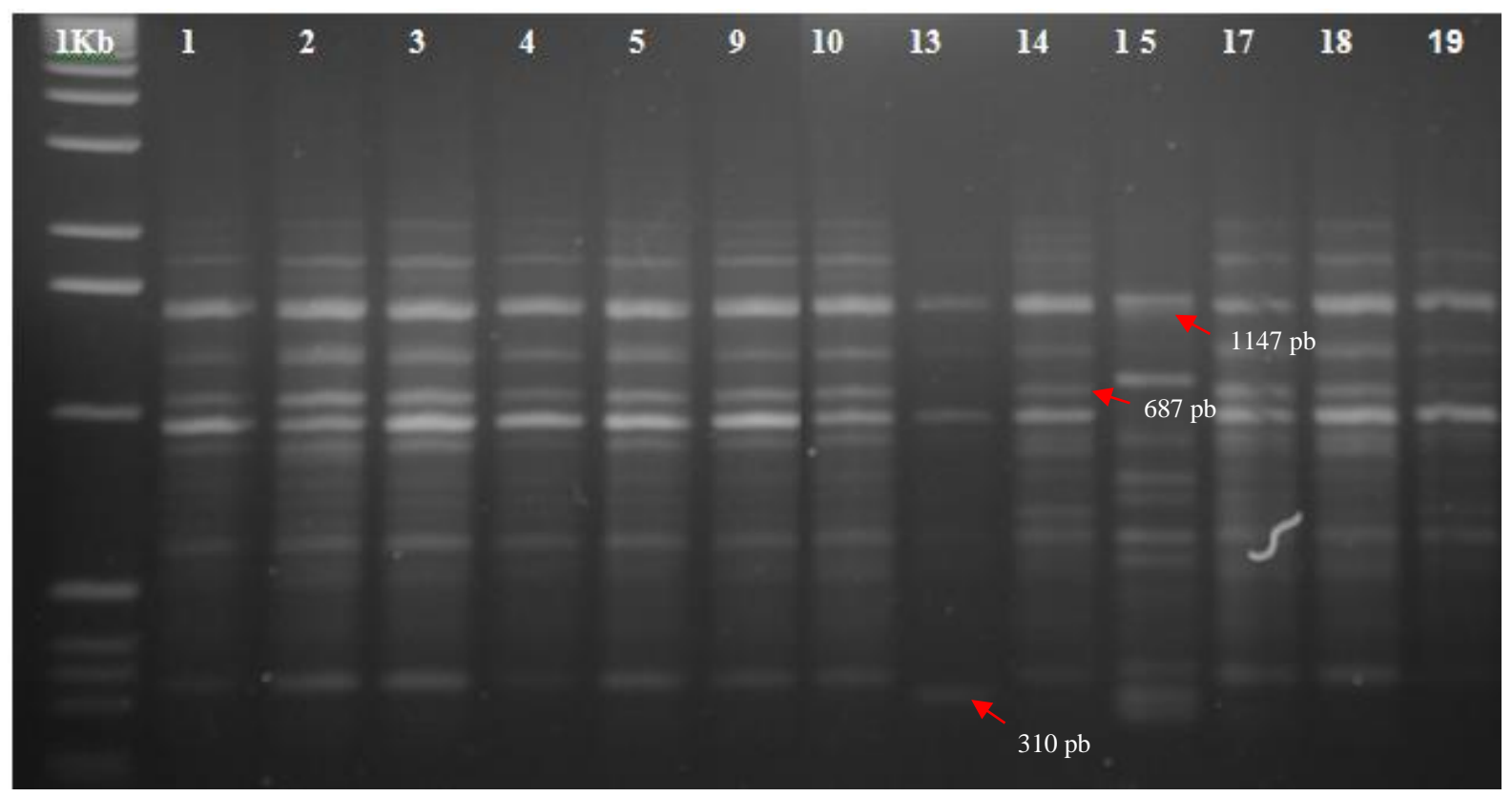

Figura 1. Patrón de bandeo generado por el iniciador SAP-04 para 13 árboles de caimito del estado de Morelos, México. $1 \mathrm{~kb}$ : marcador de peso molecular $1 \mathrm{~kb}$ DNA Ladder. 
La metodología utilizada permitió caracterizar nueve árboles, el 14 fue el que mostró mayor presencia de marcadores (30 fragmentos) mismos que fueron producidos con seis iniciadores. Esto indica que el $\mathrm{ADN}$ de este árbol tuvo mayor número de secuencias de ADN particulares que fueron complementarias a los iniciadores $\mathrm{OPB}(05,07,10,12)$, SAP-01 y SAP-04. Otro árbol que mostró mayor número de bandas marcadoras fue el árbol 15, con 15 fragmentos marcadores, generados por los oligonucleótidos OPB (06, 08, 09 y 11).

En contraste, los árboles 2, 4, 9 y 18 no produjeron ningún marcador, lo anterior significa que la molécula de ADN de estas plantas no presentó secuencias de ADN particulares que los identificaran con el uso de los iniciadores usados. Las características organolépticas de los frutos no se reportan, sin embargo, se realizó la degustación de los frutos destacando en sabor los frutos de los árboles 3, 4, 10, 14 y 15; no obstante, no se tuvieron marcadores para todos estos árboles (Cuadro 3).

Cuadro 3. Fragmentos de marcadores moleculares para 13 árboles de caimito del estado de Morelos, México.

\begin{tabular}{|c|c|}
\hline Árbol & Fragmentos marcadores \\
\hline 1 & OPB $01(1813)$ \\
\hline 2 & \\
\hline 3 & SAP 01 (669) \\
\hline 4 & \\
\hline 5 & OPB 04 (1319) \\
\hline 9 & \\
\hline 10 & OPB 05 (492), OPB $06(622,548,495)$, OPB $11(1187)$, SAP 01 (588) \\
\hline 13 & OPB $01(1906,877,1615,1044)$, OPB $07(1727)$, OPB $10(467)$, SAP $04(310,599)$ \\
\hline 14 & $\begin{array}{c}\text { OPB } 05(1339,1081,866,1265), \text { OPB } 07(2140,1396,918,496), \text { OPB } 10(2726, \\
2446,2280,1934,1762,1628,1258,899,716,654,1114), \text { OPB } 12(1192,693,596, \\
1028,665), \text { SAP } 01(1884,1689,1406,960), \text { SAP } 04(687)(306)\end{array}$ \\
\hline 15 & $\begin{array}{c}\text { OPB } 06(1877,1628,1324,1063,909,519), \text { OPB } 06(1131,995), \text { OPB } 08(1493, \\
1100,969,254,732), \text { OPB } 09(1561,1403,1940), \text { OPB } 11(987,831)\end{array}$ \\
\hline 17 & OPB $09(1357,1026,626)$, OPB $10(1208)$ \\
\hline 18 & \\
\hline 19 & OPB $04(490)$ \\
\hline
\end{tabular}

En paréntesis se indica el tamaño de fragmentos amplificados por los iniciadores correspondientes a pares de bases (pb).

La metodología RAPD permitió la caracterización de $72.9 \%$ de los árboles de caimito estudiados, esto debido a que amplifican en una amplia región del genoma; sin embargo, la posibilidad de encontrar marcadores moleculares para todos los árboles pudo ser más alta si se hubiera usado mayor cantidad de iniciadores, al respecto (Vinicius et al., 2014) al realizar un estudio para encontrar cuál era el mejor marcador para la identificación de especies de Sapotaceas, mencionan y concluyen la importancia de los marcadores moleculares, los cuales consideran son esenciales para adoptar estrategias de conservación y un plan de gestión adecuado para una especie determinada, ya que son altamente discriminatorios y útiles para la identificación molecular de la especie Sapotaceae. 
Los RAPDs en la presente investigación presentaron un alto porcentaje discriminativo, los cuales permitieron detectar la diversidad genética para la selección de árboles sobresalientes, promisorios a programas de selección, manejo y conservación que se podrían implementar eficientemente los marcadores moleculares sirven como un parámetro para clasificar y permiten identificar el material (madre) vegetal de interés a propagar.

Los fragmentos RAPDs totales y polimórficos obtenidos en la caracterización de los árboles de caimito fueron mayores a los obtenidos por Fontaine et al. (2004) quienes observaron un total de 67 loci RAPD polimórficos y 15 monomórficos que variaron de 1670 a 280 pb, en 179 individuos de la sapotaceae Vitellaria paradoxa, con los iniciadores OPB-07, OPB-11, OPN-15, OPR-15, OPW-9, OPW-12, OPW-13, OPX-3, OPX-6, OPX-11, OPY-6, OPY-13, OPY-20, OPW-5, OPW19. En esta investigación el número de bandas varió de 12 a 21, mientras que Fontaine et al. (2004), sólo identifico de 1 a 6 bandas, lo cual se atribuye a la diferencia de especies.

En cuanto al número de fragmentos marcadores, en esta investigación se obtuvieron 58 diferentes con el uso de 12 iniciadores, mientras que Heaton et al. (1999), al estudiar chicozapote mediante RAPDs con 80 iniciadores solo obtuvieron 28 bandas diferentes, esto indica la utilidad de los oligos usados en esta investigación. En esta investigación se obtuvieron 185 fragmentos polimórficos, en tanto que Degen et al. (2001), utilizando la misma metodología para estudiar 68 individuos de Chrysophyllum sanguinolentum con 11 iniciadores (OPE-02, OPE-05, OPE-07, OPY-04, OPY-06, OPY-07, OPY-10, OPY-13, OPY-14, OPY -15, OPY-16) obtuvieron sólo 48 fragmentos polimórficos. Por lo antes descrito, se puede decir que la cantidad de fragmentos amplificados varía de acuerdo con la especie a estudiar, así como a la secuencia de los indicadores.

El análisis del conglomerado obtenido (Figura 2), muestra que a un nivel de 0.71 de distancia genética se forman cuatro agrupaciones: 1) árboles 1, 4, 13, 18 y 19; 2) árboles 2, 5, 3, 10, 9 y 17; y 3) árbol 15 y 4) árbol 14. El grupo que integró más árboles fue el 2, también en este grupo se mostró la mayor similitud genética entre árboles. Las plantas dos y cinco tuvieron la menor distancia genética (0.92), en contraste los árboles 14 y 15 fueron los más distantes con respecto a los otros árboles, a la vez fueron los que mostraron mayor número de bandas marcadoras y también destacaron por sabor.

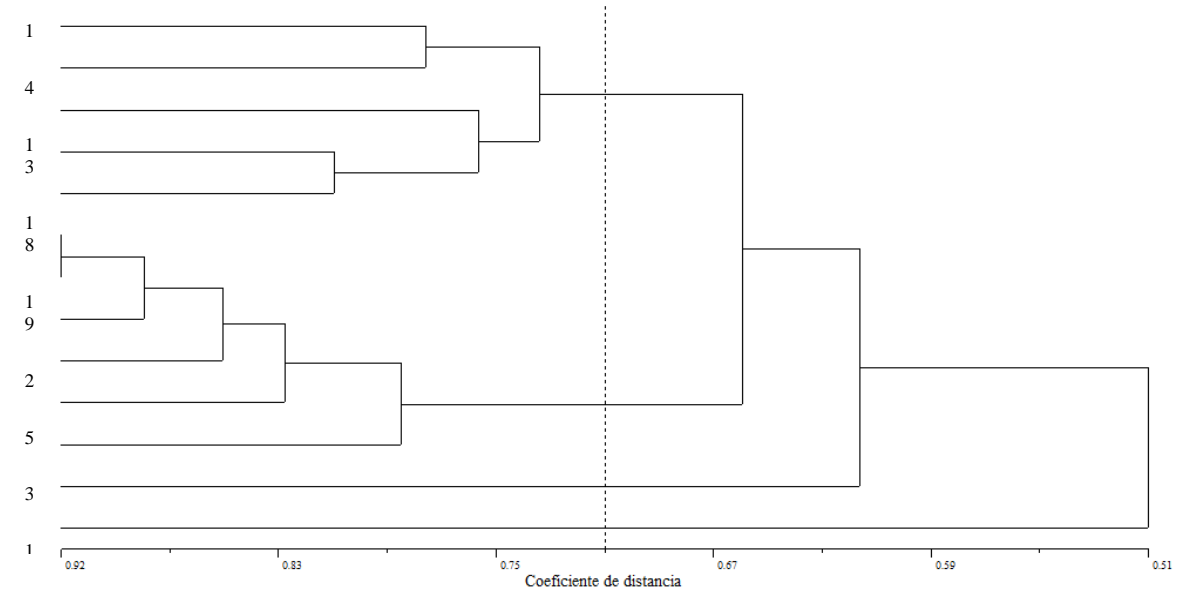

Figura 2. Dendograma de trece árboles de caimito, construido con el método de UPGMA (ligamiento promedio). El coeficiente de distancia se refiere a la distancia genética. Línea punteada: referencia para formación de grupos. 


\section{Conclusiones}

La información generada sobre la caracterización molecular del caimito es una de las primeras referencias de características de ADN, para árboles con caracteres de frutos sobresalientes, que pueden ser de utilidad para iniciar el proceso de selección y registro de variedades de esta especie frutal en México. Los RAPDs fueron de utilidad para la caracterización de nueve árboles de caimito, lográndose la caracterización molecular de más de $72.9 \%$ de las plantas estudiadas. Los árboles 14 y 15 son los que mostraron mayor número de bandas marcadoras y comparten menor similitud genética con los otros árboles.

Las especies con potencial para la conservación de germoplasma de forma in situ o ex situ son los árboles 15 y 15 los cuales pueden entrar a un programa de conservación y certificación para proporcionar a otros productores plantas con las características sobresalientes para el cultivo del caimito.

\section{Agradecimientos}

Se agradece el apoyo de PROMEP a través del proyecto UAEMOR-CA-74. Caracterización morfológica y molecular de selecciones de tres especies de sapotáceas en el estado de Morelos y al SNI (exp. 34643). A los productores de caimito del estado de Morelos por permitir el acceso a sus huertas y compartir experiencias, tiempo y convivencia.

\section{Literatura citada}

Álvarez-Vargas, J. E.; Alia-Tejacal, I.; López-Martínez, V.; Acosta-Durán, C. M.; AndradeRodríguez, M.; Colinas-León, M. T.; Delgado-Escobar, I. y Villegas-Torres, O. 2006. Caracterización de frutos de caimito (Chrysophyllum cainito L.), en el estado de Morelos. Rev. Chapingo Ser. Hortic. 12(2):217-221. https://doi.org/10.5154/r.rchsh.2006.02.019.

Alvarez, B. R. J.; Graterol, C.; Quintero, I.; Zambrano, J.; Materano, W. y Maffei, M. 2004. Evaluación de algunos métodos y prácticas de propagación en caimito (Chrysophyllum cainito L). I. Sexual. Rev. Facult. Agron. Luz. 21(4):47-53.

Anderberg, A. and Swensony, A. 2003. Evolutionary lineages in sapotaceae (Ericales): a cladistic analysis based on ndhF sequence data. Inter. J. Plant Sci. 164(21):763-773.

Andrade-Rodríguez, M.; Villegas-Monter, Á.; Gutiérrez-Espinosa, M. A.; Carrillo-Castañeda, G. and García-Velázquez, A. 2005. Polyembryony and rapd markers for identification of zygotic and nucellar seedling in citrus. Agrociencia. 39(4):371-383.

Avilán, L.; Leal, F. y Batista, D. 1992. Manual de fruticultuta: principios y manejo de la producción. Segunda edición. Editorial América, Caracas. 1387-1401 pp.

Avise, J. C. 1994. Molecular markers, natural history and evolution. Editorial Chapman and Hall. New York, 510 p.

Avise, J. C. 2004. Molecular markers, natural history and evolution. Sinauer Associates. Editorial C. and Hall. Sunderland, Massachusetts. 541 p.

Azurdia, C.; Martínez, E. y Ayala, H. 1995. Algunas sapotáceas de petén, Guatemala. Proceedings of the Interamerican Society for Tropical Horticulture. 13(1): 33-45.

Azurdia, C.; Mejia, L. y Nufio, B. 1997. Variabilidad en frutales tropicales nativos de pouteria (Sapotaceae) utilizando marcadores isoenzimáticos. Ciencia y Tecnología. 2(1):3-25. 
Bayuelo-Jiménez, J. S.; Lozano, R. J. C. y Ochoa, I. E. 2006. Caracterización morfológica de Byrsonima crassifolia (L.) kunth nativa de churumuco, Michoacán, México. Rev. Fitotec. Mex. 29(esp. 2):31-36.

Becerra, V. V. y Paredes, C. M. 2000. Uso de marcadores bioquímicos y moleculares en estudios de diversidad genética. Agric. Téc. 60(3):270-281. https://doi.org/10.4067/s036528072000000300007.

Benavides, G. A. 1998. Prospección y caracterización preliminar in situ de cinco especies de sapotaceas en Nicaragua. Repositorio Institucional La Calera, Universidad Nacional Agraria. 12-21 pp.

Borys, M. W. y Borys, H. L. 2001. El potencial genético frutícola de la República Mexicana. Editorial Fundación Salvador Sánchez Colín, Cictamex, SC. Coatepec Harinas, México. $48 \mathrm{p}$.

Carrara, S. 2004. Genetic variation among cultivated selections of mamey Sapote (Pouteria spp. [Sapotaceae]). Proc. Fla. State Hort. Soc. 117:195-200. https://doi.org/10.25148/ etd.FI14052584.

Clement, C. R. 1989. A Center of crop genetic diversity in western amazonia. Bioscience. 39(9):624-631.

Crane, J. H. and Balerdi, C. F. 2019. Caimito (star apple) growing in the florida home planting a caimito tree in the home landscape. Institute of Food and Agricultural Sciences. 1-6 pp.

Degen, B.; Caron, H.; Bandou, E.; Maggia, L.; Chevallier, M. H.; Leveau, A. and Kremer, A. 2001. Fine-scale spatial genetic structure of eight tropical tree species as analysed by RAPDs. Heredity. 87(4):497-507. https://doi.org/10.1046/j.1365-2540.2001.00942.x.

El-Hawary, S. S. E.; El-Zalabani, S. M.; Selim, N. M.; Ibrahim, M. A.; Wahba, F. A.; El-Badawy, S. A.; Mahdy, N. E. S.; Yasri, A. and Sobeh, M. 2019. Phenolic constituents of Chrysophyllum oliviforme L. Leaf down-regulate TGF- $\beta$ expression and ameliorate CCL4induced liver fibrosis: evidence from in vivo and in silico studies. Antioxidants. 8(12):646. https://doi.org/10.3390/antiox8120646.

Fontaine, C.; Lovett, P. N.; Sanou, H.; Maley, J. and Bouvet, J. M. 2004. Genetic diversity of the shea tree (Vitellaria paradoxa C. F. Gaertn), detected by RAPD and chloroplast microsatellite markers. Heredity. 93(6):639-648. https://doi.org/10.1038/sj.hdy.6800591.

González-Hernández, D.; García-Pérez, E. y Guntin-Marey, P. 2012. Caracterización genética de Manilkara zapota de Veracruz, México, con marcadores SSR. Agrociencia. 46(7):663-675.

Heaton, H. J.; Whitkus, R. and Gómez-Pompa. 1999. Extreme ecological and phenotypic differences in the tropical tree chicozapote (Manilkara zapota (L.) P. Royen) are not matched by genetic divergence: a random amplified polymorphic DNA (RAPD) analysis. Mol. Ecol. 8:627-632.

Hebert, P. and Gregory, R. 2005. Thepromise of DNA barcoding for taxonomy. Systematic Biol. 54(5):852-859.

Majourhat, K.; Jabbar, Y.; Hafidi, A. and Martínez-Gómez, P. 2008. Molecular characterization and genetic relationships among most common identified morphotypes of critically endangered rare moroccan species argania spinosa (Sapotaceae) using RAPD and SSR markers. Annal. Forest Sci. 65(8):805). https://doi.org/10.1051/forest:2008069.

Morton, J. F. 1987. Sapote. In: fruits of warm climates. Julia F. Morton, Miami. 398-402 pp.

Nascimento, E. V.; Geraldo, M. A. B. y Hissayuki, H. R. 2008. Caracterização física e química de frutos de mamey. Rev. Bras. Frutic. 30(4):953-957. https://doi.org/10.1590/s010029452008000400019. 
Orona, C. F.; Pecina, Q. V.; Rocha, P. M. A. y Cadena, H. M. A. 2006. Caracterización molecular de genotipos comerciales y elite de papa (Solanum tuberosum L.) en México. Agric. Téc. Méx. 32(2):171-180.

Parker, I. M.; López, I.; Petersen, J. J.; Anaya, A.; Cubilla-Ríos, L. and Potter, D. 2010. Domestication syndrome in Caimito (Chrysophyllum cainito L.): fruit and seed characteristics. Econ. Bot. 64(2):161-175.

Pennington, T. D. 1990. Flora neotropicana. Monografía 52. Jardín Botánico de Nueva York. $770 \mathrm{p}$.

Rennó, A. V. C.; Kanashiro, M.; Grattapaglia, D. y Yamaguishi, Y. A. 2008. Variabilidade no cpDNA em Manilkara huberi, espécie sob manejo sustentável na Amazônia brasileira. Pesqui. Agropecu. Bras. 43(7):859-867. https://doi.org/10.1590/s0100-204x2008000 700010.

Rodríguez-Rojas, T. J.; Andrade-Rodríguez, M.; Alia-Tejacal, I.; López-Martínez, V.; EspinosaZaragoza, S. y Esquinca-Avilés, H. 2012. Caracterización molecular de zapote mamey (Pouteria sapota (Jacq) Moore \& Stearn) molecular characterization of zapote mamey (Pouteria sapota (Jacq) Moore \& Stearn). Rev. Facul. Agron. Luz. 29(3):339-354.

Rojas-Rodríguez, F. y Torres-Córdoba, G. 2012. Caimito (Chrysophyllum cainito L.). Rev. Forest. Mesoam. Kurú. 9(23):45-46.

Vinicius, V. C.; Souza, M. R. C.; Alves-Araújo, A.; Marccus, A.; Mariano-Neto, E.; Den-Berg, C. and Amato, G. F. 2014. DNA barcoding in atlantic forest plants: what is the best marker for sapotaceae species identification? Gen. Mol. Biol. 37(4):662-670. https://doi.org/10.1590/S1415-47572014005000019.

Weaver, P. L. 1993. Micropholis chrysophylloides Pierra. Caimillo. Sapotaceae. Institute of Tropical Forestry, Southern Forest Experiment Station. Rio Piedras, Puerto Rico. 7 p.

Xin-hua, H.; Yong-ze, G.; Yang-mi, L. and S-jin, O. 2007. Assessment of the genetic relationship and diversity of mango and its relatives by cpISSR marker. Agric. Sci. 6(2):137-142.

Yasmin, S.; Shahidul, I.; Khondoker, N. and Samsul, A. 2006. Molecular characterization of potato germplasm by random amplified polymorphic DNA markers. Biotechnol. 5(1):27-31. https://doi.org/10.3923/biotech.2006.27.31.

Zohary, D. 1975. Unconscious selection and the evolution of domesticated plants. Econ. Bot. 58(1):5-10. https://doi.org/10.1663/0013-0001(2004)058[0005:USATEO]2.0.CO;2. 